\title{
Repurposing non-invasive prenatal testing data: Population study of single nucleotide variants associated with colorectal cancer and Lynch syndrome
}

\author{
NATALIA FORGACOVA ${ }^{1,2}$, JURAJ GAZDARICA $^{2-4}$, JAROSLAV BUDIS $^{1,3,4}$, \\ JAN RADVANSZKY ${ }^{1,2,5}$ and TOMAS SZEMES ${ }^{1-3}$
}

\begin{abstract}
${ }^{1}$ Comenius University Science Park, Comenius University; ${ }^{2}$ Department of Molecular Biology, Faculty of Natural Sciences, Comenius University; ${ }^{3}$ Geneton Ltd., 84104 Bratislava; ${ }^{4}$ Science Support Section, Slovak Centre of Scientific and Technical Information, 81104 Bratislava; ${ }^{5}$ Institute for Clinical and Translational Research, Biomedical Research Center, Slovak Academy of Sciences, 84505 Bratislava, Slovakia
\end{abstract}

Received April 29, 2021; Accepted July 16, 2021

DOI: 10.3892/ol.2021.13040

\begin{abstract}
In our previous work, genomic data generated through non-invasive prenatal testing (NIPT) based on low-coverage massively parallel whole-genome sequencing of total plasma DNA of pregnant women in Slovakia was described as a valuable source of population specific data. In the present study, these data were used to determine the population allele frequency of common risk variants located in genes associated with colorectal cancer (CRC) and Lynch syndrome (LS). Allele frequencies of identified variants were compared with six world populations to detect significant differences between populations. Finally, variants were interpreted, functional consequences were searched for and clinical significance of variants was investigated using publicly available databases. Although the present study did not identify any pathogenic variants associated with CRC or LS in the Slovak population using NIPT data, significant differences were observed in the allelic frequency of risk CRC variants previously reported in genome-wide association studies and common variants located in genes associated with LS. As Slovakia is one of the leading countries with the highest incidence of CRC among male patients in the world, there is a need for studies dedicated to investigating the cause of such a high incidence of CRC in Slovakia. The present study also assumed that extensive cross-country data aggregation of NIPT results would represent an unprecedented source of information concerning human genome variation in cancer research.
\end{abstract}

Correspondence to: Miss Natalia Forgacova, Comenius University Science Park, Comenius University, Ilkovicova 8, Karlova Ves, 84104 Bratislava, Slovakia

E-mail: natali.forgacova@gmail.com

Key words: colorectal cancer, Lynch syndrome, non-invasive prenatal testing, low-coverage massively parallel whole genome sequencing

\section{Introduction}

Colorectal cancer (CRC) is the third most common cancer worldwide and the second most common cancer in Europe, causing an estimated 9.4\% of all cancer deaths in Europe. $\mathrm{CRC}$ is also a serious societal problem in Slovakia, with an incidence rate of $15,7 \%$ and a mortality rate of $15,6 \%[(1,2)$ GLOBOCAN 2020 data]. Many risk factors and causes are associated with the likelihood of developing CRC, but the main reason is still not fully understood. The considerable geographical variability suggests that $\mathrm{CRC}$ is a complex polygenic disease caused by genetic and environmental factors and their interactions. Age, sex, lifestyle, and dietary habits $(3,4)$, including meat and alcohol consumption $(5,6)$, tobacco smoking (7-9), obesity and lack of physical activity $(4,10,11)$ play a major role in the pathogenesis of CRC. Other well-known risk factors may also be inflammatory bowel diseases, acromegaly, renal transplantation with long-term immunosuppression, diabetes mellitus and insulin resistance, cholecystectomy, or androgen deprivation therapy $(3,4)$.

Beside these, inherited susceptibility plays a significant role in the etiology of CRC because it can be responsible for about $35 \%$ of all cases of colorectal cancer. However, high-penetrance germline variants in known genes $(A P C$, BRCA2, KRAS, NTS, SMAD4, POLE, BRAF, BMPRIA, POLD1, STK11, MUTYH and DNA mismatch repair genes), which are associated with severe hereditary syndromes, such as familial adenomatous polyposis and Lynch syndrome (also called hereditary non-polyposis colorectal cancer), account for only $5-7 \%$ of total CRC cases $(4,12)$. Therefore, the remaining unknown heritability is probably explained by the interaction of common, low-penetrance variants identified through genome-wide association studies (GWAS). GWAS in ethnic/racial minority populations offers the opportunity to uncover genetic susceptibility factors and discover new genomic regions and loci that contribute risk for CRC development. Since 2007, more than 100 common risk variants have been successfully identified in GWAS, which have helped to elucidate the etiology of CRC (13-18). 
Lynch syndrome (LS) is an autosomal dominant hereditary cancer syndrome that accounts for approximately $3 \%$ of all colorectal cancer cases (19). From a clinical point of view, $10-82 \%$ (20) of LS cases are associated with a lifetime risk of developing CRC, unless the risk is significantly lower in other types of cancer $(21,22)$. LS is caused by pathogenic germline mutations in a class of genes called DNA mismatch repair (MMR) genes, mainly $M L H 1$, located in 3p22.2 chromosome, and $M S H 2$, located in 2p21 chromosome (23), which represent $70-85 \%$ of cases of LS (24). Mutations found in MSH6 (2p16.3), PMS2 (7p22.1) (25) and MLH3 genes have lower incidence (26). Molecular investigations have also shown that MSH3 (27) and germline 3'deletions of the EPCAM gene, which lead to epigenetic silencing of $M S H 2$ (28), are also implicated in the pathogenesis of LS. As a consequence of MMR pathway inactivation and loss of expression of MMR proteins, DNA replication errors accumulate typically resulting in microsatellite instability (MSI), which is generally detected in LS patients' tumor tissues (29). The diagnosis of LS involves three main steps, identification of patients and their familial history that meet the Amsterdam or Bethesda guidelines, presence of MSI in tumors and immunohistochemical analysis (IHC) of MMR protein expression. A definitive diagnosis of LS must be confirmed by detecting the germline mutations in MMR genes (30).

Non-invasive prenatal testing (NIPT) based on low-coverage massively parallel whole-genome sequencing of plasma DNA from pregnant women generates a large amount of data that provides the resources to investigate human genetic variations in the population. In our previous studies, we described the re-use of the data from NIPT for genome-scale population specific frequency determination of small DNA variants (31) and CNVs (32). Since pregnant women represent a relatively standard sample of the local female population, we assumed this NIPT data could also be used in the population study of CRC, the most common cancer in Slovakia. Some research concerning on genomic analysis of plasma from NIPT has also demonstrated NIPT data's efficiency and utility for viral genetic studies (33), genetic profiling of Vietnamese population (34) or detection of CNV aberrations (32,35).

The main aim of our study was a detailed analysis of common variants (MAF $>0,05$ ) that showed evidence of association with CRC in GWAS datasets and characterization of population variability from data generated by NIPT. We assumed that the genetic factors, mainly the increased specific population frequency of CRC and LS variants could be responsible for the high incidence of CRC in Slovakia. To test this hypothesis, allele frequencies of risk CRC variants identified in the Slovak population were compared with allele frequencies of risk CRC variants in 6 worldwide populations. As LS is among the most common hereditary CRC syndromes, the aim of our study was also to analyze population allele frequencies and describe clinical impacts of relevant variants located in known LS predisposing genes. To our knowledge, this was the first population study of CRC using NIPT data conducted exclusively in the Slovak population.

\section{Materials and methods}

Data source. The laboratory procedure used, to generate the NIPT data, were as follows: DNA from plasma of peripheral maternal blood was isolated for NIPT analysis from 1,501 pregnant women after obtaining a written informed consent consistent with the Helsinki declaration from the subjects. The population cohort consisted from women in reproductive age between 17-48 years with a median of 35 years. Genomic information from a sample consisted of maternal and fetal DNA fragments. Each included individual agreed to use their genomic data in an anonymized form for general biomedical research. The NIPT study (study ID 35900_2015) was approved by the Ethical Committee of the Bratislava Self-Governing Region (Sabinovska ul.16, 82005 Bratislava) on 30th April of 2015 under the decision ID 03899_2015. Blood samples were collected to EDTA tubes and plasma was separated in dual centrifugation procedure. DNA was isolated from $700 \mu \mathrm{l}$ of plasma using DNA Blood Mini kit (Qiagen) according to standard protocol. Sequencing libraries were prepared from each sample using TruSeq Nano kit HT (Illumina) following standard protocol with omission of DNA fragmentation step. Each sample was normalized to $4 \mathrm{~nm}$ library and the final concentration of libraries was 2,8 pM. Individual barcode labelled libraries were pooled and sequenced using low-coverage whole-genome sequencing on an Illumina NextSeq500 platform (Illumina) by performing paired end sequencing of 2x35 bases (36).

Data analysis. The datasets generated and/or analyzed during the current study are available in the DSpace repository, https://dspace.uniba.sk/xmlui/handle/123456789/27 (31).

Analyses of common variants previously reported to be risk variants for $C R C$. We combined genotype data from all previously reported GWAS studies available online (https://www.gwascentral.org/) for the years 2007-2020, specifically 66 GWAS studies of CRC risk variants that included individuals with European, Asian and African American ancestry. Using data from these GWAS datasets, we identified 116 risk variants associated with CRC, which were then merged with our data of identified variants from NIPT. Risk variants that were not found in NIPT data were excluded from the analysis. All identified variants in the Slovak population used for further analyses were common (MAFs $>0.05$ ). Subsequently, allele frequencies of CRC risk variants for each population (East Asian, South Asian, African, American, Finnish European and non-Finnish European) were extracted from the gnomAD database available online (v3.0, downloaded from https:/gnomad.broadinstitute.org/downloads) and compared with our frequencies determined for the Slovak population from NIPT data. Allele frequency in each population and allele frequency differences were plotted using boxplots. Outliers of boxplots that represent variants with highly different frequencies between Slovak and non-Finnish populations were annotated via published literature and studies [in dbSNP (https://www.ncbi.nlm.nih.gov/snp/) and GWAS (https://www. gwascentral.org/)]. To assess the relations between allele frequency of CRC risk variants in each population, we also used Principal Component Analysis (PCA) using matplotlib. pyplot library, which reduces the dimension of the data to a graphically interpretable 2D or 3D dimension. Consequently, we obtained information on which populations have similar or different allele frequencies of the identified CRC risk variants. 
Analyses of variants located in genes associated with LS. After analyzing variants associated with $\mathrm{CRC}$, we focused on the study of variants associated with LS. First, we filtered out a group of variants located in 7 genes known to be associated with LS (MLH1, PMS2, MSH6, MLH3, MSH2, TGFBR2, EPCAM). The genomic locations of genes were determined by the GeneCards database (https://www.genecards.org/). From the dataset of identified variants in LS associated genes, we excluded variants in low complexity genomic regions (soft masked in the reference FASTA file), eliminating the variants that could represent sequencing artifacts or repetitive regions. All variants that were used for further analysis were annotated using Ensembl Variant Effect Predictor (VEP, version 101_GRCh38). In our dataset, based on ClinVar database annotation of the most common types of pathogenic and likely pathogenic variants associated with LS (https://www.ncbi.nlm. nih.gov/clinvar), we selected variants including frameshift, missense, nonsense, splice site, non-coding and UTR variants. After this filtering, allele frequencies for both groups of variants (all variants identified in LS genes and selected types of variants) for each population (East Asian, South Asian, African, American, Finnish European and non-Finnish European) were extracted from the gnomAD database (v3.0, downloaded from https:/gnomad.broadinstitute.org/downloads) and compared with our frequencies determined for the Slovak population from NIPT data. Allele frequency in each population and allele frequency differences were plotted using boxplots and PCA analysis using matplotlib.pyplot library. Outliers of boxplots representing variants with allele frequency differences more than $10 \%$ were annotated via published literature and studies [in dbSNP (https://www.ncbi.nlm.nih.gov/snp/) and GWAS (https://www.gwascentral.org/].

\section{Results}

Analyses of common variants previously reported to be risk variants for $C R C$. In the analysis of the 66 GWAS studies that included all identified risk variants associated with colorectal carcinogenesis from 2007-2020, we investigated 116 variants. There were 25 independent CRC risk variants in Asian population, 62 risk variants found in European population, 27 risk variants in both European and Asian population and 2 risk variants located in African American population that were previously reported in GWAS (Table I).

After merging all identified variants from GWAS (116 risk variants) with our NIPT data, we identified 106 common risk CRC variants (Table SI), while 10 risk variants that were not called in the Slovak population were excluded from further analysis. The allele frequencies of 106 variants identified in our population sample (Slovak population) and the allele frequencies of variants for 6 world populations (East Asian, South Asian, African, American, Finnish European and non-Finnish European) obtained by gnomAD database (Table SII) are shown in graphical comparison by Boxplots (Fig. 1) and principal component analysis (PCA) (Fig. 2). As shown in Fig. 1, the MAF ranged from 0.0-0.963109 in 6 world populations that is comparable with the Slovak population (0.0521-0.931). The median allele frequency for the Slovak population reached the value of 0.4072 , which is closest to the value of the median of the American population $(\mathrm{MED}=0.3991)$ and Finnish population $(\mathrm{MED}=0.4166)$. PCA placed our sample set most closely to the two gnomAD population sample sets, i.e., to the Finnish and non-Finnish European population.

Next, we compared known allele frequencies of 106 CRC risk variants in our sample set from the Slovak population to allele frequencies of CRC variants in six world populations. The final findings of allele frequency differences are shown in Fig. 3. The median allele frequency for comparing the Slovak population and non-Finnish European population reached the value of 0.002285 . Together, we identified 14 outliers in Fig. 3 (3 of 14 variants reached a similar value and overlapped, so they are not clearly visible in Fig. 3). Since the same variant rs4246215 was identified in 4 different population comparisons (Slovak-American, Slovak-Finnish European, Slovak-non-Finnish European, and also identified in Slovak-East Asian population comparison) and the rs3131043 variant in 2 population comparisons (Slovak-Finnish European and Slovak-non-Finnish European population comparison), we identified a total of 10 variants, whose difference in population allele frequency was more than $10 \%$. Table II includes annotation information about these variants by dbSNP NCBI, ClinVar database, and population comparison in which they were identified.

Analyses of variants located in genes associated with LS. In the analysis of LS, we identified 1212 variants in our sample set from NIPT that were located in genes known to be associated with LS, i.e., MLH1, PMS2, MSH6, TGFBR2, MLH3, MSH2 and $E P C A M$. After excluding variants from low complexity regions, we obtained 648 variants that were finally annotated by VEP and used for further analysis. The allele frequencies of 648 variants identified in our population sample (Slovak population) and the allele frequencies of variants for 6 world populations (East Asian, South Asian, African, American, Finnish European and non-Finnish European) obtained by gnomAD database (Table SIII) are shown in graphical comparison by Boxplots (Fig. 4) and principal component analysis (PCA) (Fig. 5). As shown in Fig. 4, the MAF ranged from 0.0-1.0 in 6 world populations. In the Slovak population, all variants were with MAF>0.05 (0.0502-1.0). The median allele frequency for the Slovak population reached the value of 0.2204 , which is closest to the value of the median of the South Asian population $(\mathrm{MED}=0.221274)$. $\mathrm{PCA}$ placed our sample set most closely to the non-Finnish European population (Fig. 5).

In the next step, to identify variants having significantly different frequencies, we compared known allele frequencies of 648 variants located in genes associated with LS identified in our sample set from the Slovak population to allele frequencies of these variants in six gnomAD world populations. The final findings of allele frequency differences are shown in Fig. 6. The median allele frequency for the comparison of the Slovak population and non-Finnish European population reached the value of -0.01093 . By comparing the allele frequency of variants of the Slovak and non-Finnish populations, we identified a total of 64 outliers. Most outliers were found in the MSH2 gene, others in MSH6, TGFBR2, PMS2, MLH1 and EPCAM. We did not identify any outlying variant in the $M L H 3$ gene. The variation type of all outliers was 'intronic variant' and the clinical significance of all outliers was not reported 
Table I. Identification of 116 risk variants associated with colorectal cancer from 66 genome-wide association studies between 2007 and 2020.

\begin{tabular}{|c|c|c|c|c|c|c|c|}
\hline First author, year & rs_ID & $\mathrm{Chr}$ & POS & POP & Gene & PMID & (Refs.) \\
\hline Wang et al, 2017 & rs 7252505 & $19 q 13$ & $33,084,158$ & AFR A & GPATCH1 & 28295283 & (46) \\
\hline Wang et al, 2017 & rs56848936 & $19 q 13.3$ & $46,321,507$ & AFR A & SYMPK & 28295283 & (46) \\
\hline Lu et al, 2019 & rs7542665 & $1 \mathrm{p} 31.3$ & $62,673,037$ & ASN & L1TD1 & 30529582 & (13) \\
\hline Law et al, 2019 & rs12143541 & $1 \mathrm{p} 32.3$ & $55,247,852$ & ASN & TTC22 & 31089142 & (43) \\
\hline Lu et al, 2019 & rs201395236 & $1 q 44$ & $245,181,421$ & ASN & EFCAB2 & 30529582 & (13) \\
\hline Lu et al, 2019 & rs7606562 & $2 \mathrm{p} 16.3$ & $48,686,695$ & ASN & PPP1R21 & 30529582 & (13) \\
\hline Lu et al, 2019 & rs 113569514 & $3 q 22.2$ & $133,748,789$ & ASN & SLCO2A1 & 30529582 & (13) \\
\hline Lu et al, 2019 & rs 12659017 & $5 q 23.2$ & $125,988,175$ & ASN & $\begin{array}{l}\text { ALDH7A1, } \\
\text { PHAX }\end{array}$ & 30529582 & (13) \\
\hline Law et al, 2019 & rs639933 & $5 q 31.1$ & $134,467,751$ & ASN & $\begin{array}{l}\text { C5orf66, } \\
\text { LOC } 105379188\end{array}$ & 31089142 & (43) \\
\hline Jia et al, 2013 & rs647161 & $5 q 31.1$ & $134,499,092$ & ASN & PITX1 & 23263487 & $(58)$ \\
\hline Law et al, 2019 & rs6933790 & $6 \mathrm{p} 21.1$ & $41,672,769$ & ASN & TFEB & 31089142 & $(43)$ \\
\hline Zeng et al, 2016 & rs4711689 & $6 \mathrm{p} 21.1$ & $41,692,812$ & ASN & TFEB & 26965516 & (14) \\
\hline Schmit et al, 2018 & rs6906359 & $6 \mathrm{p} 21.31$ & $35,528,378$ & ASN & FKBP5 & 29917119 & (18) \\
\hline Lu et al, 2019 & rs3830041 & $6 \mathrm{p} 21.32$ & $32,191,339$ & ASN & NOTCH4 & 30529582 & (13) \\
\hline Lu et al, 2019 & rs6584283 & $10 \mathrm{q} 24.2$ & $101,290,301$ & ASN & NKX2-3 & 30529582 & (13) \\
\hline Lu et al, 2019 & rs77969132 & $12 \mathrm{p} 11.21$ & $31,594,813$ & ASN & DENND5B & 30529582 & (13) \\
\hline Zeng et al, 2016 & rs11064437 & $12 \mathrm{p} 13.31$ & $6,982,162$ & ASN & SPSB2 & 26965516 & (14) \\
\hline Lu et al, 2019 & rs2730985 & $12 q 12$ & $43,130,624$ & ASN & PRICKLE1 & 30529582 & (13) \\
\hline Lu et al, 2019 & rs 1886450 & $13 q 22.1$ & $73,986,628$ & ASN & KLF5, KLF12 & 30529582 & (13) \\
\hline Lu et al, 2019 & rs4341754 & $16 \mathrm{q} 23.2$ & $80,039,621$ & ASN & WWOX, MAF & 30529582 & (13) \\
\hline Lu et al, 2019 & rs 1078643 & $17 \mathrm{p} 12$ & $10,707,241$ & ASN & PIRT & 30529582 & (13) \\
\hline Law et al, 2019 & rs 73975588 & $17 \mathrm{p} 13.3$ & 816,741 & ASN & $\mathrm{NXN}$ & 31089142 & (43) \\
\hline Law et al, 2019 & rs9797885 & $19 q 13.2$ & $41,873,001$ & ASN & TMEM91 & 31089142 & (43) \\
\hline Law et al, 2019 & rs6055286 & 20p12.3 & $7,718,045$ & ASN & None & 31089142 & (43) \\
\hline Jia et al, 2008 & rs2423279 & $20 p 12.3$ & $7,812,350$ & ASN & HAO1 & 23263487 & $(58)$ \\
\hline Law et al, 2019 & rs2179593 & $20 \mathrm{q} 13.12$ & $42,660,286$ & ASN & TOX2 & 31089142 & $(43)$ \\
\hline Lu et al, 2019 & rs 13831 & $20 q 13.32$ & $57,475,191$ & $\mathrm{ASN}$ & GNAS & 30529582 & (13) \\
\hline Law et al, 2019 & rs61776719 & $1 \mathrm{p} 34.3$ & $38,461,319$ & EUR & None & 31089142 & (43) \\
\hline Peters et al, 2013 & rs 10911251 & $1 \mathrm{q} 25.3$ & $183,112,059$ & EUR & LAMC1 & 23266556 & $(59)$ \\
\hline Whiffin et al, 2014 & rs 10911251 & $1 \mathrm{q} 25.3$ & $183,112,059$ & EUR & LAMC1 & 24737748 & $(60)$ \\
\hline Houlston et al, 2010 & rs6687758 & $1 q 41$ & $222,164,948$ & EUR & None & 20972440 & (61) \\
\hline Houlston et al, 2010 & rs6691170 & $1 q 41$ & $222,045,446$ & EUR & DUSP10 & 20972440 & (61) \\
\hline Law et al, 2019 & rs 11692435 & $2 q 11.2$ & $98,275,354$ & EUR & ACTR1B & 31089142 & (43) \\
\hline Law et al, 2019 & rs11893063 & $2 q 33.1$ & $199,601,925$ & EUR & LOC105373831 & 31089142 & (43) \\
\hline Law et al, 2019 & rs7593422 & $2 q 33.1$ & $200,131,695$ & EUR & None & 31089142 & $(43)$ \\
\hline Orlando et al, 2016 & rs992157 & $2 q 35$ & $219,154,781$ & EUR & TMBIM1 & 27005424 & $(62)$ \\
\hline Law et al, 2019 & rs9831861 & $3 \mathrm{p} 21.1$ & $53,088,285$ & EUR & None & 31089142 & $(43)$ \\
\hline Law et al, 2019 & rs 12635946 & $3 q 13.2$ & $112,916,918$ & EUR & None & 31089142 & (43) \\
\hline Houlston et al, 2010 & rs 10936599 & $3 q 26.2$ & $169,774,313$ & EUR & MYNN & 20972440 & $(61)$ \\
\hline Schmit et al, 2018 & rs 1370821 & $4 q 22.2$ & $94,943,383$ & EUR & None & 29917119 & (18) \\
\hline Law et al, 2019 & rs 17035289 & $4 q 24$ & $106,048,291$ & EUR & None & 31089142 & $(43)$ \\
\hline Law et al, 2019 & rs75686861 & 4q31.21 & $145,621,328$ & EUR & HHIP & 31089142 & (43) \\
\hline Schmit et al, 2014 & rs35509282 & $4 q 32.2$ & $163,333,405$ & EUR & FSTL5 & 25023989 & (63) \\
\hline
\end{tabular}


Table I. Continued.

\begin{tabular}{|c|c|c|c|c|c|c|c|}
\hline First author, year & rs_ID & $\mathrm{Chr}$ & POS & POP & Gene & PMID & (Refs.) \\
\hline Schmit et al, 2018 & rs58791712 & $5 \mathrm{p} 13.1$ & $40,281,797$ & EUR & PTGER4 & 29917119 & (18) \\
\hline Schmit et al, 2018 & rs 2735940 & $5 p 15.33$ & $1,296,486$ & EUR & TERT & 29917119 & $(18)$ \\
\hline Peters et al, 2012 & rs2853668 & $5 \mathrm{p} 15.33$ & 1299910 & EUR & TERT & 21761138 & (64) \\
\hline Schmit et al, 2018 & rs62404968 & $6 \mathrm{p} 12.1$ & $55,714,314$ & EUR & BMP5 & 29917119 & $(18)$ \\
\hline Dunlop et al, 2012 & rs 1321311 & $6 \mathrm{p} 21.2$ & $36,622,900$ & EUR & CDKN1A & 22634755 & $(48)$ \\
\hline Law et al, 2019 & rs9271770 & $6 \mathrm{p} 21.32$ & $32,594,248$ & EUR & LOC107987449 & 31089142 & (43) \\
\hline Law et al, 2019 & rs3131043 & $6 \mathrm{p} 21.33$ & $30,758,466$ & EUR & HCG20 & 31089142 & (43) \\
\hline Law et al, 2019 & rs2070699 & $6 \mathrm{p} 24.1$ & $12,292,772$ & EUR & EDN1 & 31089142 & (43) \\
\hline Law et al, 2019 & rs6928864 & $6 q 21$ & $105,966,894$ & EUR & None & 31089142 & (43) \\
\hline Law et al, 2019 & rs 10951878 & $7 \mathrm{p} 12.3$ & $46,926,695$ & EUR & None & 31089142 & (43) \\
\hline Law et al, 2019 & rs3801081 & $7 \mathrm{p} 12.3$ & $47,511,161$ & EUR & TNS3 & 31089142 & $(43)$ \\
\hline Tomlinson et al, 2008 & rs 16892766 & $8 q 23.3$ & $117,630,683$ & EUR & EIF3H & 18372905 & (64) \\
\hline Law et al, 2019 & rs 1412834 & $9 p 21.3$ & $22,110,131$ & EUR & CDKN2B-AS1 & 31089142 & $(43)$ \\
\hline Schmit et al, 2018 & rs 10994860 & $10 q 11.23$ & $52,645,424$ & EUR & $\mathrm{A} 1 \mathrm{CF}$ & 29917119 & (18) \\
\hline Al Tassan et al, 2015 & rs 10904849 & $10 \mathrm{p} 13$ & $16,955,267$ & EUR & CUBN & 25990418 & $(15)$ \\
\hline Law et al, 2019 & rs4450168 & $11 \mathrm{p} 15.4$ & $10,286,755$ & EUR & $\mathrm{SBF} 2$ & 31089142 & $(43)$ \\
\hline Dunlop et al, 2012 & rs3824999 & 11q13.4 & $74,345,550$ & EUR & POLD3 & 22634755 & $(48)$ \\
\hline Tenesa et al, 2008 & rs3802842 & 11q23.1 & $111,171,709$ & EUR & COLCA2 & 18372901 & (66) \\
\hline Houlston et al, 2010 & rs7136702 & $12 q 13.12$ & $50,880,216$ & EUR & LARP4 & 20972440 & (61) \\
\hline Law et al, 2019 & rs7398375 & $12 q 13.3$ & $57,540,848$ & EUR & LRP1 & 31089142 & $(43)$ \\
\hline Schmit et al, 2018 & rs72013726 & $12 \mathrm{q} 24.21$ & $115,890,835$ & EUR & MED13L & 29917119 & (18) \\
\hline Schmit et al, 2018 & rs 10161980 & $13 q 13.2$ & $34,093,518$ & EUR & STARD13 & 29917119 & $(18)$ \\
\hline Law et al, 2019 & rs 12427600 & $13 q 13.3$ & $37,460,648$ & EUR & SMAD9 & 31089142 & (43) \\
\hline Law et al, 2019 & rs45597035 & $13 q 22.1$ & $73,649,152$ & EUR & KLF5 & 31089142 & (43) \\
\hline Law et al, 2019 & rs1330889 & $13 q 22.3$ & $78,609,615$ & EUR & LINC00446 & 31089142 & (43) \\
\hline Law et al, 2019 & rs7993934 & $13 q 34$ & $111,074,915$ & EUR & COL4A2 & 31089142 & (43) \\
\hline Tomlinson et al, 2011 & rs 1957636 & $14 q 22.2$ & $54,560,018$ & EUR & BMP4 & 21655089 & $(67)$ \\
\hline Houlston et al, 2008 & rs4444235 & $14 q 22.2$ & $54,410,919$ & EUR & BMP4 & 19011631 & $(68)$ \\
\hline Tomlinson et al, 2011 & rs4444235 & $14 q 22.2$ & $54,410,919$ & EUR & BMP4 & 21655089 & $(67)$ \\
\hline Tomlinson et al, 2008 & rs 11632715 & $15 q 13.3$ & $33,004,247$ & EUR & None & 18372905 & $(65)$ \\
\hline Tomlinson et al, 2008 & rs16969681 & $15 q 13.3$ & $32,993,111$ & EUR & SCG5 & 18372905 & $(65)$ \\
\hline Tomlinson et al, 2011 & rs16969681 & $15 q 13.3$ & $32,993,111$ & EUR & SCG5 & 21655089 & (67) \\
\hline Tomlinson et al, 2008 & rs4779584 & $15 q 13.3$ & $32,994,756$ & EUR & CRAC1 & 18372905 & $(65)$ \\
\hline Law et al, 2019 & rs4776316 & $15 q 22.31$ & $67,007,813$ & EUR & SMAD6 & 31089142 & (43) \\
\hline Law et al, 2019 & rs 10152518 & $15 q 23$ & $68,177,162$ & EUR & None & 31089142 & $(43)$ \\
\hline Law et al, 2019 & rs7495132 & $15 q 26.1$ & $91,172,901$ & EUR & CRTC3 & 31089142 & (43) \\
\hline Houlston et al, 2008 & rs9929218 & $16 q 22.1$ & $68,820,946$ & EUR & $\mathrm{CDH} 1$ & 19011631 & $(68)$ \\
\hline Law et al, 2019 & rs61336918 & $16 q 23.2$ & $80,007,266$ & EUR & None & 31089142 & (43) \\
\hline Schmit et al, 2018 & rs2696839 & $16 \mathrm{q} 24.1$ & $86,340,448$ & EUR & FOXF1 & 29917119 & $(18)$ \\
\hline Broderick et al, 2007 & rs4939827 & $18 \mathrm{q} 21$ & $46,453,463$ & EUR & SMAD7 & 17934461 & (69) \\
\hline Tenesa et al, 2008 & rs4939827 & $18 \mathrm{q} 21$ & $46,453,463$ & EUR & SMAD7 & 18372901 & $(66)$ \\
\hline Law et al, 2019 & rs285245 & $19 \mathrm{p} 13.11$ & $16,420,817$ & EUR & None & 31089142 & (43) \\
\hline Houlston et al, 2008 & rs 10411210 & $19 q 13.11$ & $33,532,300$ & EUR & RHPN2 & 19011631 & $(68)$ \\
\hline Law et al, 2019 & rs12979278 & $19 q 13.33$ & $49,218,602$ & EUR & MAMSTR & 31089142 & $(43)$ \\
\hline Tomlinson et al, 2011 & rs4813802 & $20 p 12.3$ & $6,699,595$ & EUR & BMP2 & 21655089 & (67) \\
\hline
\end{tabular}


Table I. Continued.

\begin{tabular}{|c|c|c|c|c|c|c|c|}
\hline First author, year & rs_ID & Chr & POS & POP & Gene & PMID & (Refs.) \\
\hline Peters et al, 2012 & rs4813802 & $20 \mathrm{p} 12.3$ & $6,699,595$ & EUR & BMP2 & 21761138 & (64) \\
\hline Houlston et al, 2008 & rs961253 & 20p12.3 & $6,404,281$ & EUR & BMP2 & 19011631 & $(68)$ \\
\hline Schmit et al, 2018 & rs2295444 & $20 q 11.22$ & $33,173,883$ & EUR & PIGU & 29917119 & $(18)$ \\
\hline Schmit et al, 2018 & rs 1810502 & $20 q 13.13$ & $49,057,488$ & EUR & PTPN1 & 29917119 & $(18)$ \\
\hline Law et al, 2019 & rs3787089 & $20 q 13.33$ & $62,316,630$ & EUR & RTEL1 & 31089142 & $(42)$ \\
\hline Houlston et al, 2010 & rs4925386 & $20 q 13.33$ & $60,921,044$ & EUR & LAMA5 & 20972440 & $(61)$ \\
\hline Schumacher et al, 2015 & rs8124813 & $3 p 14.1$ & $43,476,841$ & EUR, ASN & LRIG1 & 26151821 & $(70)$ \\
\hline Schumacher et al, 2015 & rs 35360328 & $3 p 22.1$ & $40,924,962$ & EUR, ASN & CTNNB1 & 26151821 & $(70)$ \\
\hline Lu et al, 2019 & rs 1476570 & $6 \mathrm{p} 22.1$ & $29,809,860$ & EUR, ASN & HLA-G & 30529582 & (13) \\
\hline Tomlinson et al, 2008 & rs2450115 & $8 q 23.3$ & $117,624,093$ & EUR, ASN & EIF3H & 18372905 & $(67)$ \\
\hline Tomlinson et al, 2008 & rs6469656 & $8 q 23.3$ & $117,647,788$ & EUR, ASN & EIF3H & 18372905 & $(67)$ \\
\hline Haiman et al, 2007 & rs6983267 & $8 \mathrm{q} 24.21$ & $128,413,305$ & EUR, ASN & POU5F1B & 17618282 & (71) \\
\hline Tomlinson et al, 2007 & rs6983267 & $8 \mathrm{q} 24.21$ & $128,413,305$ & EUR, ASN & POU5F1B & 17618284 & (72) \\
\hline Hutter et al, 2010 & rs6983267 & $8 \mathrm{q} 24.21$ & $128,413,305$ & EUR, ASN & POU5F1B & 21129217 & $(73)$ \\
\hline Cui et al, 2011 & rs6983267 & $8 \mathrm{q} 24.21$ & $128,413,305$ & EUR, ASN & POU5F1B & 21242260 & (4) \\
\hline Law et al, 2019 & rs 12255141 & $10 \mathrm{q} 25.2$ & $114,294,892$ & EUR, ASN & VTI1A & 31089142 & (43) \\
\hline Zhang et al, 2014 & rs704017 & $10 q 22.3$ & $80,819,132$ & EUR, ASN & ZMIZ1 & 24836286 & (16) \\
\hline Whiffin et al, 2014 & rs 1035209 & $10 q 24.2$ & $101,345,366$ & EUR, ASN & SLC25A28 & 24737748 & $(60)$ \\
\hline Zeng et al, 2016 & rs4919687 & $10 \mathrm{q} 24.32$ & $104,595,248$ & EUR, ASN & CYP17A1 & 26965516 & (14) \\
\hline Zhang et al, 2014 & rs11196172 & $10 \mathrm{q} 25.2$ & $114,726,843$ & EUR, ASN & TCF7L2 & 24836286 & (16) \\
\hline Wang et al, 2014 & rs 12241008 & $10 q 25.2$ & $114,280,702$ & EUR, ASN & VTI1A & 25105248 & $(75)$ \\
\hline Zhang et al, 2014 & rs 1535 & 11q12.2 & $61,597,972$ & EUR, ASN & FADS2 & 24836286 & (16) \\
\hline Zhang et al, 2014 & rs 174550 & $11 \mathrm{q} 12.2$ & $61,571,478$ & EUR, ASN & FADS1 & 24836286 & (16) \\
\hline Zhang et al, 2014 & rs4246215 & $11 \mathrm{q} 12.2$ & $61,564,299$ & EUR, ASN & FEN1 & 24836286 & $(16)$ \\
\hline Zhang et al, 2014 & rs 174537 & $11 \mathrm{q} 12.2$ & $61,552,680$ & EUR, ASN & MYRF & 24836286 & (16) \\
\hline Law et al, 2019 & rs 10849438 & $12 \mathrm{p} 13.31$ & $6,412,036$ & EUR, ASN & None & 31089142 & $(43)$ \\
\hline Zhang et al, 2014 & rs 10849432 & $12 \mathrm{p} 13.31$ & $6,385,727$ & EUR, ASN & PLEKHG6 & 24836286 & (16) \\
\hline Peters et al, 2013 & rs3217810 & $12 \mathrm{p} 13.32$ & $4,388,271$ & EUR, ASN & CCND2 & 23266556 & $(59)$ \\
\hline Whiffin et al, 2014 & rs3217810 & $12 \mathrm{p} 13.32$ & $4,388,271$ & EUR, ASN & CCND2 & 24737748 & $(60)$ \\
\hline Jia et al, 2013 & rs 10774214 & $12 \mathrm{p} 13.32$ & $4,368,352$ & EUR, ASN & CCND2 & 23263487 & $(58)$ \\
\hline Zhang et al, 2014 & rs 12603526 & $17 \mathrm{p} 13.3$ & 800,593 & EUR, ASN & $\mathrm{NXN}$ & 24836286 & (16) \\
\hline Zhang et al, 2014 & rs7229639 & $18 q 21.1$ & $46,450,976$ & EUR, ASN & SMAD7 & 24836286 & (16) \\
\hline Zhang et al, 2014 & rs 1800469 & $19 q 13.2$ & $41,860,296$ & EUR, ASN & TMEM91 & 24836286 & (16) \\
\hline Zhang et al, 2014 & rs2241714 & $19 q 13.2$ & $41,869,392$ & EUR, ASN & B9D2 & 24836286 & $(16)$ \\
\hline Schumacher et al, 2015 & rs606682520 & $20 \mathrm{q} 13.13$ & 897,353 & EUR, ASN & PREX1 & 26498495 & $(70)$ \\
\hline Schumacher et al, 2015 & rs6066825 & $20 q 13.13$ & $47,340,117$ & EUR, ASN & PREX1 & 26151821 & $(70)$ \\
\hline Dunlop et al, 2012 & rs5934683 & Xp22.2 & $9,751,474$ & EUR, ASN & SHROOM2 & 22634755 & $(48)$ \\
\hline
\end{tabular}

Chr, chromosome; POS, position; POP, population; AFR A, African American; ASN, Asian; EUR, European.

in ClinVar. All this annotation information, also including chromosome position, variants ID, reference and alternative allele, genes, is available in Table SIV.

Our analysis also included allele frequency comparison of selected variants from 648 variants identified in our sample set of NIPT data. We focused on frameshift, missense, nonsense, splice site, non-coding and UTR variants, annotated in the ClinVar database as the most common types of pathogenic and likely pathogenic variants associated with LS. However, from these selected types of variants, we found only UTR 


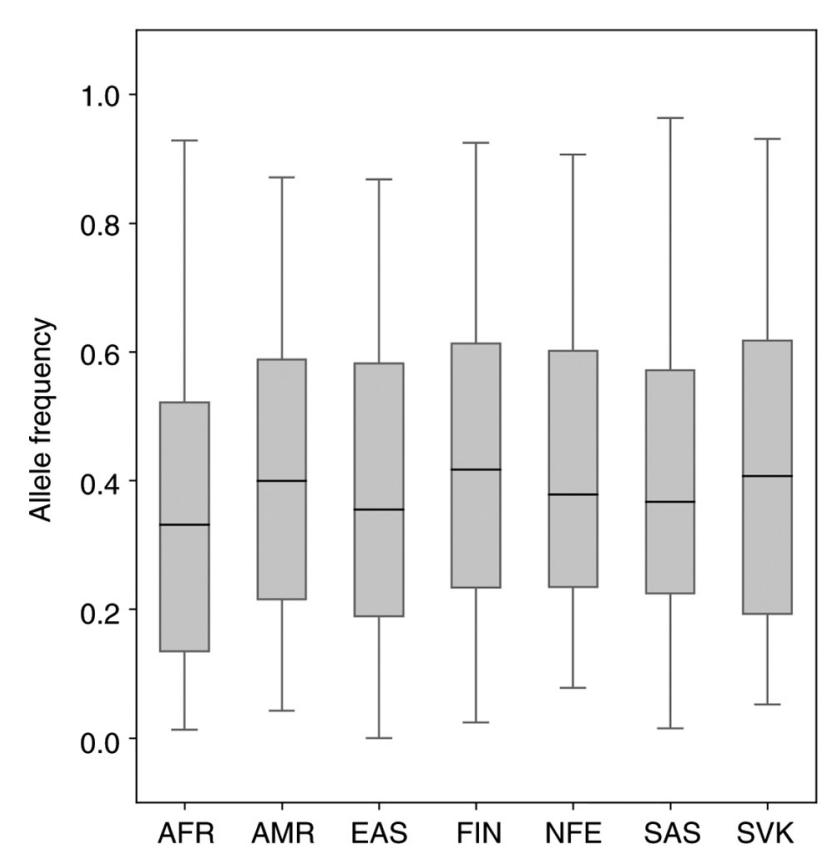

Figure 1. Boxplots show allele frequency of 106 risk colorectal cancer variants identified from genome-wide association studies for the Slovak and other six world populations. AFR, African population; AMR, American population; EAS, East Asian population; FIN, European (Finnish) population; NFE, European (non-Finnish) population; SAS, South Asian population; SVK, Slovak population.

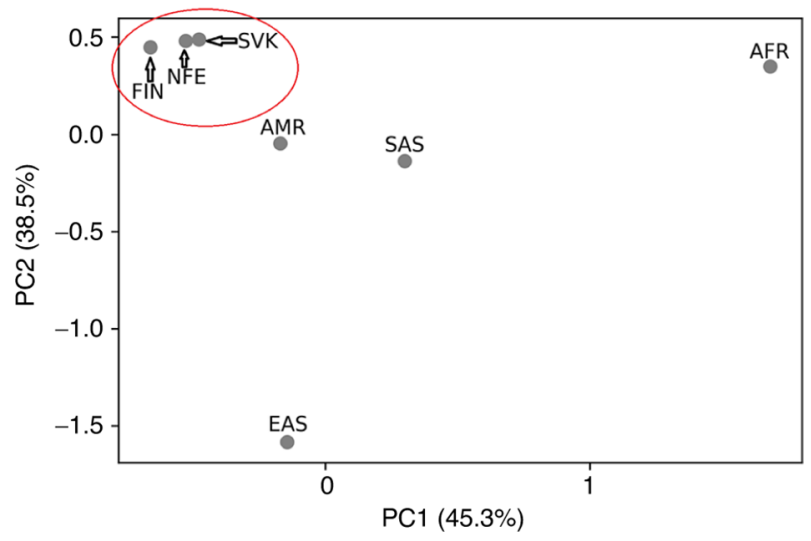

Figure 2. Principal Component Analysis plot illustrates the allele frequency of 106 risk colorectal cancer variants identified from genome-wide association studies for the Slovak and other six world populations. PC1, Principal Component 1; PC2, Principal Component 2; AFR, African population AMR, American population; EAS, East Asian population; FIN, European (Finnish) population; NFE, European (non-Finnish) population; SAS, South Asian population; SVK, Slovak population.

and non-coding variants in our dataset of 648 variants. Other types of variants (downstream, upstream, and intron) were excluded from further analysis. Finally, we selected 18 variants, 10 UTR and 8 non-coding variants (all selected variants with annotation information by VEP and ClinVar are available in Table III). We compared known allele frequencies of these 18 selected variants identified in our population sample (Slovak population) to the six gnomAD world populations. The final findings of allele frequency differences are shown in Fig. 7. The median allele frequency for the comparison of the Slovak population and non-Finnish

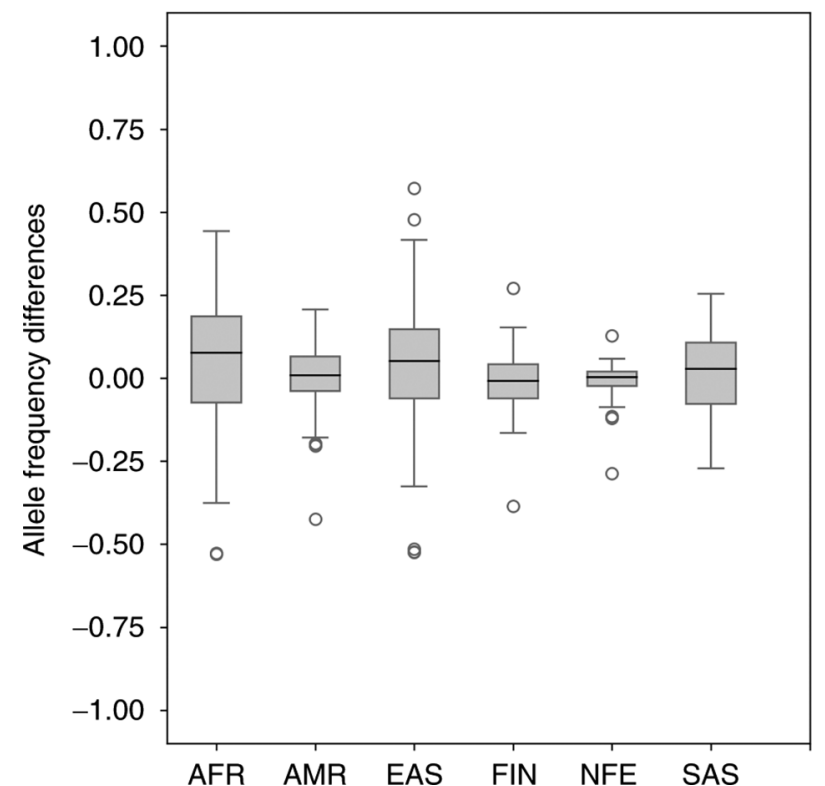

Figure 3. Boxplots show allele frequency differences of Slovak and other six world populations for 106 risk colorectal cancer variants identified from genome-wide association studies. AFR, African population; AMR, American population; EAS, East Asian population; FIN, European (Finnish) population; NFE, European (non-Finnish) population; SAS, South Asian population.

European population reached the value of 0.014215 , which is closest to the value of the median allele frequency comparison of the South Asian and Slovak population (MED=0.014186). By comparing the allele frequency of variants of the Slovak and six gnomAD world population, we identified a total of 4 outliers-rs10951973, rs10951972 (identified in Slovak-American population comparison), rs6791557 (in Slovak-American and Slovak-non-Finnish European population comparison) and rs9852378 (in Slovak-South Asian population comparison). All outliers were non-coding variants, rs10951973 and rs10951972 located in the PMS2 and rs6791557 located in the TGFBR2 were not reported in ClinVar. The rs9852378 SNP, detected in the $M L H 1$, was reported as benign by ClinVar.

Finally, we analyzed allele frequencies of pathogenic and likely pathogenic variants associated with Lynch syndrome annotated in the ClinVar database. From 229 SNPs with pathogenic and likely pathogenic clinical significance, only 15 have non-zero AF records in the gnomAD database. As shown in Fig. 8, all found AF are significantly below 5\% (Table IV and Fig. 8).

\section{Discussion}

Population genetic studies currently have a huge impact on the study of genomics (37). The detection of risk variants in a population and identifying their genetic relationships have advanced our understanding of the human genome's variability and led to the elucidation of many factors that influence cancer risk. In recent years, NGS technologies have played a key role in colorectal cancer research and have become a useful tool for cancer diagnostics and screening (38-41). Due to the high incidence of colorectal cancer in the Slovak population, it is 
Table II. Outliers identified in boxplots that show allele frequency differences of Slovak and the other six world populations for 106 risk colorectal cancer variants identified from genome-wide association studies.

\begin{tabular}{|c|c|c|c|c|c|c|c|}
\hline rs_ID & $\begin{array}{l}\text { Population } \\
\text { comparison }\end{array}$ & $\mathrm{Chr}$ & POS & $\begin{array}{l}\text { Variant } \\
\text { type }\end{array}$ & Gene & Consequence & Clinical significance \\
\hline rs5934683 & Slovak-East Asian & $\operatorname{chr} \mathrm{X}$ & 9783434 & SNV & GPR143 & Intron Variant & Not Reported in ClinVar \\
\hline rs7252505 & Slovak-African & $\operatorname{chr} 19$ & 33084158 & SNV & GPATCH1 & Intron Variant & Not Reported in ClinVar \\
\hline rs4779584 & Slovak-East Asian & $\operatorname{chr} 15$ & 32702555 & SNV & None & None & Not Reported in ClinVar \\
\hline rs 174550 & Slovak-American & $\operatorname{chr} 11$ & 61804006 & SNV & FADS1 & Intron Variant & Not Reported in ClinVar \\
\hline \multirow[t]{4}{*}{ rs4246215 } & Slovak-American & chr11 & 61796827 & SNV & FEN1 & 3' UTR Variant & Not Reported in ClinVar \\
\hline & $\begin{array}{l}\text { Slovak-European } \\
\text { (Finnish) }\end{array}$ & $\operatorname{chr} 11$ & 61796827 & SNV & FEN1 & 3' UTR Variant & Not Reported in ClinVar \\
\hline & $\begin{array}{l}\text { Slovak-European } \\
\text { (non-Finnish) }\end{array}$ & $\operatorname{chr} 11$ & 61796827 & SNV & FEN1 & 3' UTR Variant & Not Reported in ClinVar \\
\hline & Slovak-East Asian & $\operatorname{chr} 11$ & 61796827 & SNV & FEN1 & 3' UTR Variant & Not Reported in ClinVar \\
\hline rs10904849 & $\begin{array}{l}\text { Slovak-European } \\
\text { (non-Finnish) }\end{array}$ & $\operatorname{chr} 10$ & 16955267 & SNV & CUBN & Intron Variant & Not Reported in ClinVar \\
\hline rs6928864 & Slovak-African & chr6 & 105519019 & SNV & None & None & Not Reported in ClinVar \\
\hline \multirow[t]{2}{*}{ rs3131043 } & $\begin{array}{l}\text { Slovak-European } \\
\text { (non-Finnish) }\end{array}$ & chr6 & 30790689 & SNV & HCG20 & Intron Variant & Not Reported in ClinVar \\
\hline & $\begin{array}{l}\text { Slovak-European } \\
\text { (Finnish) }\end{array}$ & chr6 & 30790689 & SNV & HCG20 & Intron Variant & Not Reported in ClinVar \\
\hline rs12659017 & Slovak-East Asian & $\operatorname{chr} 5$ & 126652483 & SNV & None & None & Not Reported in ClinVar \\
\hline rs397775554 & $\begin{array}{l}\text { Slovak-European } \\
\text { (non-Finnish) }\end{array}$ & $\operatorname{chr} 5$ & $\begin{array}{r}40281696- \\
40281704\end{array}$ & Indel & None & None & Not Reported in ClinVar \\
\hline
\end{tabular}

Chr, chromosome; POS, position; SNV, single nucleotide variant.

crucial to determine the possible causes of the high incidence of this disease in Slovakia.

Non-invasive prenatal testing of common fetal chromosomal aberrations, using low-coverage massively parallel whole-genome sequencing of maternal plasma cfDNA of pregnant women, has become the fastest low-cost genomic DNA test that is rapidly implemented in clinical practice. Currently, more than 3 million NIPT tests are carried out worldwide each year, and the large amount of data generated during NIPT provides the resources to investigate human genetic variations in the population (31). In our study, we analyzed low-coverage massively parallel whole-genome sequencing data of total plasma DNA from pregnant women generated for NIPT screening to characterize the variants in genes associated with CRC and LS in the Slovak population. To our knowledge, the present study is the first population analysis of CRC and LS variants worldwide and also in the Slovak population using NIPT data. We illustrate the utility of these genomic data for clinical genetics and population studies.

Over the past two decades, GWAS offer the opportunity to uncover genetic susceptibility factors for CRC and provide insights into the biological basis of CRC etiology. These studies have demonstrated that only a fraction of CRC heritability is explained by known risk-conferring genetic variation, whereas the remaining genetic risk of CRC may be accounted for by a combination of high-prevalence and low-penetrance of common genetic variants. To date, a large number of common genetic variants have been identified by the GWAS approach, which has intimately connected to the onset of CRC $(13,18,42-46)$.

By pooling GWAS data of risk variants associated with colorectal carcinogenesis from 2007-2020 and data variants in our population sample from NIPT, we have identified 106 common risk $\mathrm{CRC}$ variants. When we compared allele frequencies of these variants to allele frequencies in six gnomAD world population, finally 13 common risk variants were found that showed statistically significant differences in population allele frequencies-rs5934683, rs7252505, rs4779584, rs1535, rs174550, rs4246215, rs11196172, rs10904849, rs6928864, rs3131043, rs1476570, rs12659017, rs397775554.

The SNP rs5934683 is located on chromosome Xp22.2 between two genes, GPR143 (G protein-coupled receptor 143), which is expressed by melanocytes and retinal pigment epithelium and SHROOM2 (shroom family member 2), a human homolog of the Xenopus laevis APX gene that has important functions in cell morphogenesis including endothelial and epithelial tissue development (44). Missense mutations in this gene have been detected in large-scale screens for recurring mutations in cancer cell lines. Both GPR143 and SHROOM2 play a role in melanosome biogenesis and retinal pigmentation. It is known that abnormal retinal pigmentation, similar to the congenital hypertrophy of retinal pigment epithelium lesions, are typical of the familial adenomatous polyposis syndrome (FAP), one of the inherited syndromes of CRC (47). The relationship between Xp22.2 and CRC risk represents the first evidence 


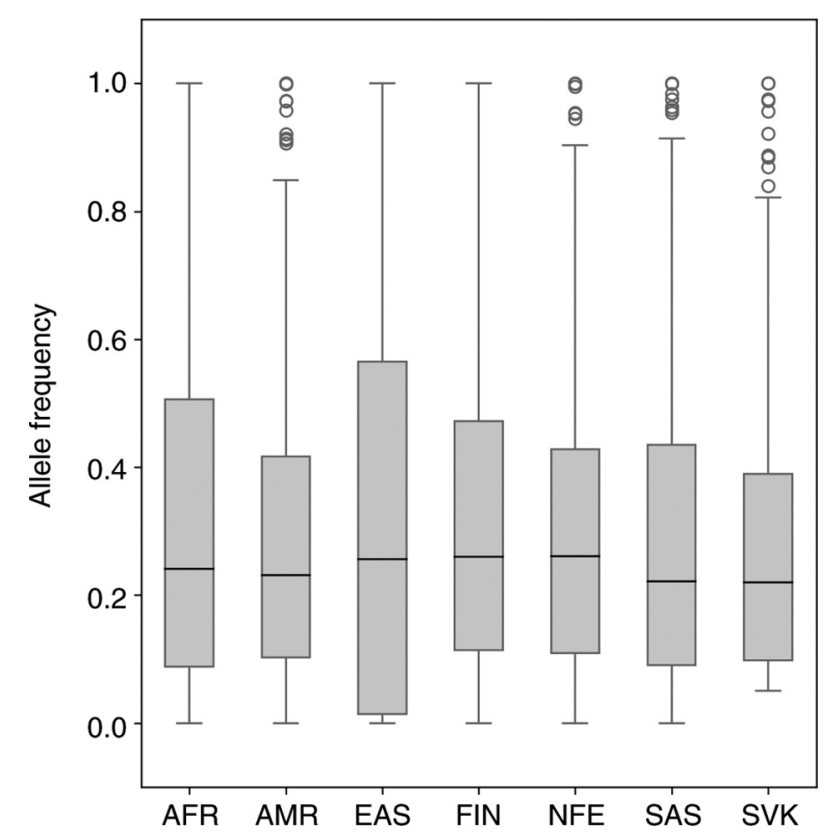

Figure 4. Boxplots show allele frequency of 648 variants located in seven Lynch syndrome genes for the Slovak and other six world populations. AFR, African population; AMR, American population; EAS, East Asian population; FIN, European (Finnish) population; NFE, European (non-Finnish) population; SAS, South Asian population; SVK, Slovak population.

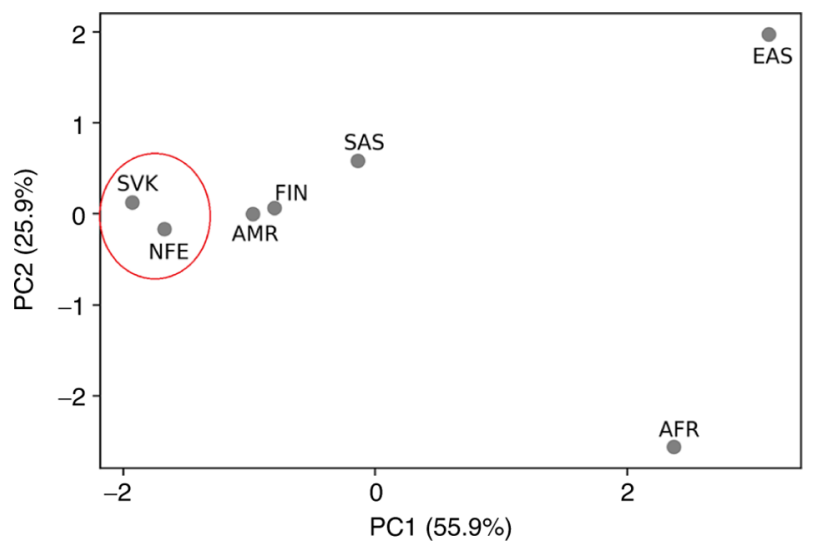

Figure 5. Principal Component Analysis plot illustrates the allele frequency of 648 variants located in genes associated with Lynch syndrome for the Slovak and other six world populations. PC1, Principal Component 1; PC2, Principal Component 2; AFR, African population; AMR, American population; EAS, East Asian population; FIN, European (Finnish) population; NFE, European (non-Finnish) population; SAS, South Asian population; SVK, Slovak population.

for the role of $\mathrm{X}$-chromosome variation in predisposition to non-sex-specific cancer (48).

The SNP rs7252505, located in the 19q13 risk locus, is in an intron of the gene GPATCH1 (G-patch domain containing 1). Although GPATCH1 is expressed in the colon, little is known about its function other than the fact that it contains a G-patch domain, a domain typically associated with RNA processing. One study found that rs7252505 was associated with CRC in African Americans $(46,49)$.

Intergenic variant rs4779584 in chromosomal region $15 \mathrm{q} 13.3$ lies between SCG5 and GREM1, and the association

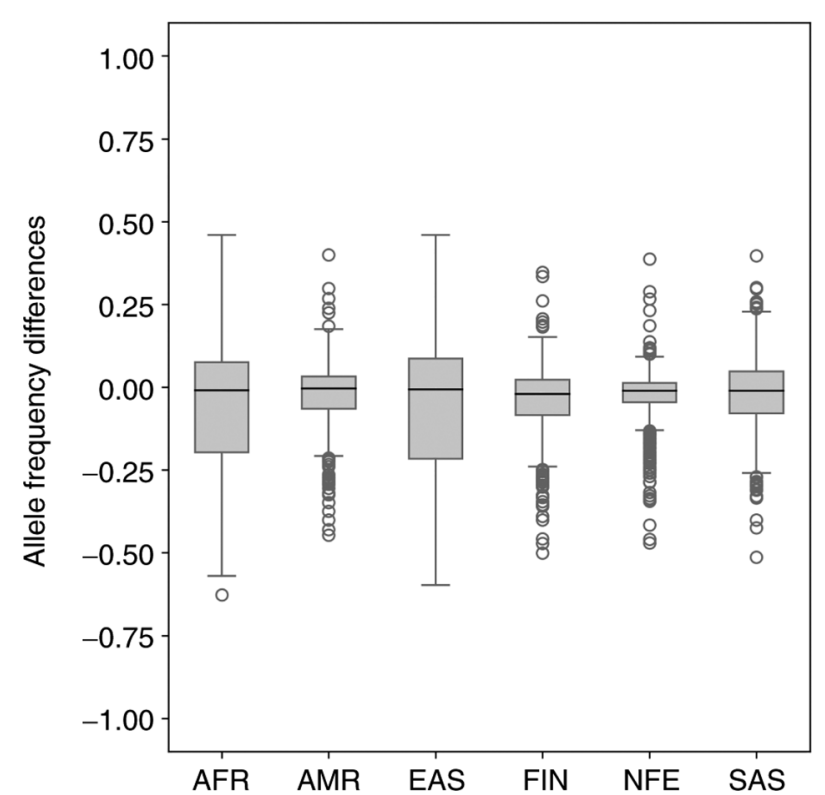

Figure 6. Boxplots show differences of Slovak and the other six world populations in allele frequency for 648 variants located in genes associated with Lynch syndrome. AFR, African population; AMR, American population; EAS, East Asian population; FIN, European (Finnish) population; NFE, European (non-Finnish) population; SAS, South Asian population.

between this SNP to CRC has been identified in several GWAS studies $(13,50)$.

The rs4246215 polymorphism is located in the FEN1 in the long arm of chromosome 11 (11q12.2). The association between this SNP and the potential risk of different types of cancers, including esophageal, lung, gastrointestinal, gallbladder, breast cancer in Chinese and Iran populations, glioma and childhood leukemia, has been previously studied. The rs4246215 variant was also associated with colorectal cancer in East Asians and the Chinese population $(51,52)$.

To identify variants that may predispose to LS and may cause the high incidence of CRC in Slovakia, we used NIPT data, including variants with at least 5\% AF and coverage at least 100 reads per variant. To verify the reliability of the found variants using NIPT, we selected 15 variants with AF below $5 \%$ and validated them using Sanger sequencing. For this reason, it is not possible to find rare variants with $\mathrm{AF}$ under $5 \%$. Initially, we selected gene variants known to be associated with LS and we focused on their population AF in gnomAD database and as well as on pathogenicity as reported in public database ClinVar. No publications are available for all variants showing statistically significant differences in population allele frequencies and selected 18 variants. The rs9852378 SNP was reported as benign by ClinVar, and other variants were not reported in ClinVar.

Our study has several key shortcomings. None of the variants identified in this study are pathogenic or likely pathogenic due to their extremely low frequency in the general population (Fig. 8). From the total number of pathogenic or likely pathogenic variants annotated in the ClinVar database, we could determine the population frequency of only 15 variants even when using the gnomAD database (Table IV). Second, the sample size was relatively small and it is strongly biased towards females. We assume 
Table III. Identification of 18 selected variants (UTR and non-coding) from all 648 variants in genes associated with Lynch syndrome from non-invasive prenatal testing data in the Slovak population.

\begin{tabular}{lccclcc}
\hline rs_ID & Chr & POS & Variant type & Gene & Consequence & Clinical significance \\
\hline rs11901645 & chr2 & 47510079 & SNV & MSH2 & 3' UTR variant & Not reported in ClinVar \\
rs11891189 & chr2 & 47510259 & SNV & MSH2 & 3' UTR variant & Not reported in ClinVar \\
rs876936 & chr2 & 47513059 & SNV & MSH2 & 3' UTR variant & Not reported in ClinVar \\
rs72872839 & chr2 & 47565070 & SNV & MSH2 & 3' UTR variant & Not reported in ClinVar \\
rs17036769 & chr2 & 47633503 & SNV & MSH2 & 3' UTR variant & Not reported in ClinVar \\
rs2969774 & chr2 & 47661684 & SNV & MSH2 & 3' UTR variant & Not reported in ClinVar \\
rs2952372 & chr2 & 47661919 & SNV & MSH2 & 3' UTR variant & Not reported in ClinVar \\
rs2969773 & chr2 & 47662141 & SNV & MSH2 & 3' UTR variant & Not reported in ClinVar \\
rs2705765 & chr2 & 47662389 & SNV & MSH2 & 3' UTR variant & Not reported in ClinVar \\
rs12328344 & chr2 & 47662542 & SNV & MSH2 & 3' UTR variant & Not reported in ClinVar \\
rs10427209 & chr2 & 47709297 & SNV & MSH6 & Non-coding transcript exon variant & Not reported in ClinVar \\
rs10427344 & chr2 & 47709476 & SNV & MSH6 & Non-coding transcript exon variant & Not reported in ClinVar \\
rs3136240 & chr2 & 47784947 & SNV & MSH6 & Non-coding transcript exon variant & Not reported in ClinVar \\
rs6791557 & chr3 & 30614676 & SNV & TGFBR2 & Non-coding transcript exon variant & Not reported in ClinVar \\
rs1817338 & chr3 & 30631239 & SNV & TGFBR2 & Non-coding transcript exon variant & Not reported in ClinVar \\
rs9852378 & chr3 & 36997280 & SNV & MLH1 & Non-coding transcript exon variant & Benign in ClinVar \\
rs10951972 & chr7 & 6002187 & SNV & PMS2 & Non-coding transcript exon variant & Not reported in ClinVar \\
rs10951973 & chr7 & 6002205 & SNV & PMS2 & Non-coding transcript exon variant & Not reported in ClinVar \\
\hline
\end{tabular}

Chr, chromosome; POS, position; SNV, single nucleotide variant.

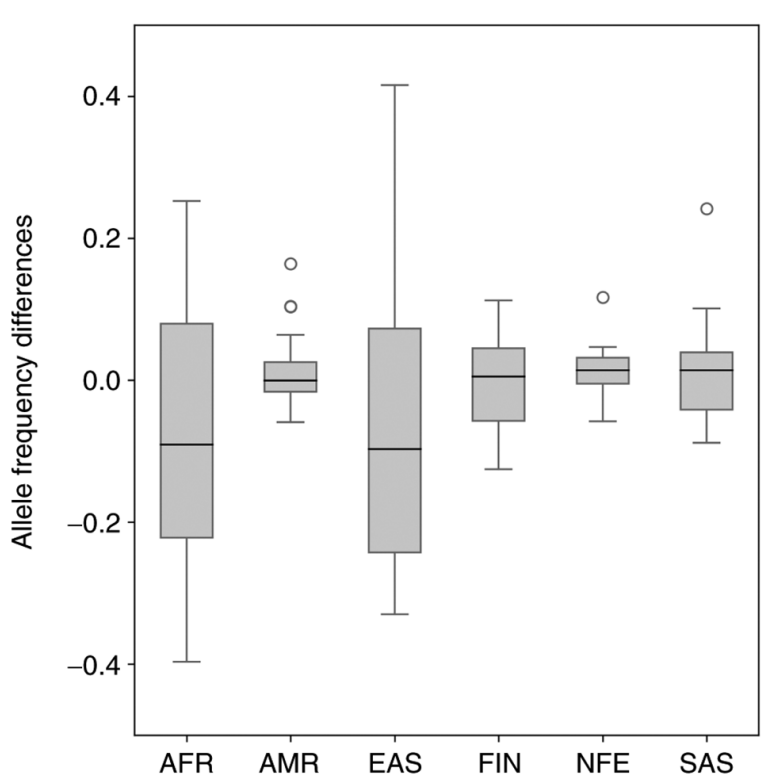

Figure 7. Boxplots show differences of Slovak and the other six gnomAD world populations in allele frequency for 18 selected variants (UTR and non-coding variants) from 648 identified variants located in Lynch syndrome risk genes. AFR, African population; AMR, American population; EAS, East Asian population; FIN, European (Finnish) population; NFE, European (non-Finnish) population; SAS, South Asian population.

that even larger sample sets will also not offer opportunities to detect such low frequencies of LS variants in the population using NIPT data. Third, a substantial portion of identified variants was removed from analyses due to technical limitations, mainly because of their location in

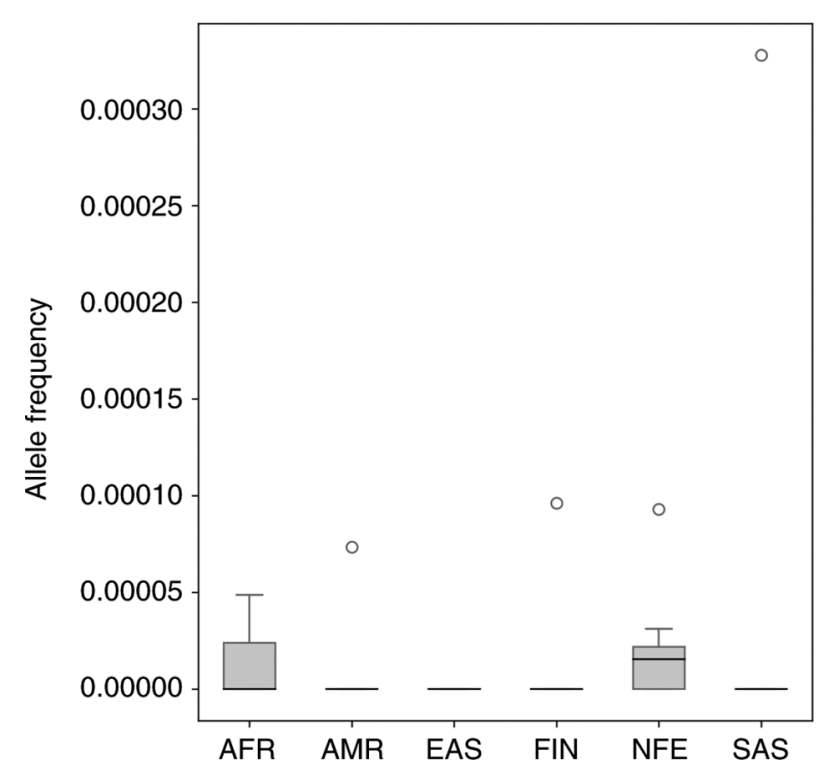

Figure 8. Boxplots show allele frequency of 15 single nucleotide polymorphisms of Lynch syndrome genes with pathogenic and likely pathogenic clinical significance for six world populations. It was found that allele frequency was $<5 \%$. AFR, African population; AMR, American population; EAS, East Asian population; FIN, European (Finnish) population; NFE, European (non-Finnish) population; SAS, South Asian population.

low complexity regions. Although these could be technical artifacts (53), they could also be real variants having biological effects that are yet generally hardly determinable, but likely existing (54). Moreover, colorectal cancer is a disease caused by a combination of multiple genes and 
Table IV. Identification of 15 pathogenic and likely pathogenic variants associated with Lynch syndrome with non-zero allele frequency in gnomAD database.

Allele frequency in population

\begin{tabular}{|c|c|c|c|c|c|c|c|c|c|c|}
\hline \multirow[b]{2}{*}{ rs_ID } & \multirow[b]{2}{*}{ Chr } & \multirow[b]{2}{*}{ POS } & \multirow[b]{2}{*}{$\begin{array}{l}\text { REF } \\
\text { Allele }\end{array}$} & \multirow[b]{2}{*}{$\begin{array}{c}\text { ALT } \\
\text { Allele }\end{array}$} & & & & & & \\
\hline & & & & & African & American & $\begin{array}{l}\text { East } \\
\text { Asian }\end{array}$ & $\begin{array}{l}\text { European } \\
\text { (Finnish) }\end{array}$ & $\begin{array}{c}\text { European } \\
\text { (non-Finnish) }\end{array}$ & $\begin{array}{l}\text { South } \\
\text { Asian }\end{array}$ \\
\hline rs63750615 & 2 & 47403333 & G & $\mathrm{T}$ & 0 & 0 & 0 & 0 & 0 & $3.28 \times 10^{-5}$ \\
\hline rs1194793421 & 2 & 47414417 & $\mathrm{AG}$ & $\mathrm{A}$ & 0 & 0 & 0 & 0 & $2.75 \times 10^{-5}$ & 0 \\
\hline rs63750636 & 2 & 47476492 & $\mathrm{C}$ & $\mathrm{T}$ & 0 & 0 & 0 & 0 & $1.55 \times 10^{-5}$ & 0 \\
\hline rs63749873 & 2 & 47795903 & $\mathrm{C}$ & $\mathrm{G}$ & 0 & 0 & 0 & 0 & $3.10 \times 10^{-5}$ & 0 \\
\hline rs587783056 & 2 & 47799684 & GTT & $\mathrm{G}$ & $4.76 \times 10^{-5}$ & 0 & 0 & 0 & 0 & 0 \\
\hline rs63751017 & 2 & 47800714 & $\mathrm{C}$ & $\mathrm{T}$ & 0 & 0 & 0 & 0 & $3.10 \times 10^{-5}$ & 0 \\
\hline rs876660943 & 2 & 47806359 & G & $\mathrm{T}$ & 0 & 0 & 0 & 0 & $1.55 \times 10^{-5}$ & 0 \\
\hline rs63751221 & 3 & 37001045 & $\mathrm{C}$ & $\mathrm{T}$ & $2.38 \times 10^{-5}$ & 0 & 0 & 0 & 0 & 0 \\
\hline rs587779338 & 7 & 5977589 & G & A & $4.86 \times 10^{-5}$ & 0 & 0 & 0 & $1.58 \times 10^{-5}$ & 0 \\
\hline rs267608161 & 7 & 5982885 & $\mathrm{C}$ & $\mathrm{T}$ & $4.80 \times 10^{-5}$ & 0 & 0 & $9.61 \times 10^{-5}$ & $1.55 \times 10^{-5}$ & 0 \\
\hline rs63751422 & 7 & 5986838 & $\mathrm{G}$ & A & $2.38 \times 10^{-5}$ & 0 & 0 & 0 & 0 & 0 \\
\hline rs63750250 & 7 & 5986933 & A & AT & 0 & 0 & 0 & 0 & $9.30 \times 10^{-5}$ & 0 \\
\hline rs200640585 & 7 & 5992018 & $\mathrm{G}$ & A & 0 & 0 & 0 & 0 & $1.55 \times 10^{-5}$ & 0 \\
\hline rs267608154 & 7 & 5995572 & ACTGT & A & 0 & 0 & 0 & 0 & $1.55 \times 10^{-5}$ & 0 \\
\hline rs63750871 & 7 & 6002590 & G & A & 0 & $7.33 \times 10^{-5}$ & 0 & 0 & 0 & 0 \\
\hline
\end{tabular}

Chr, chromosome; POS, position; REF, reference; ALT, alternative.

environmental factors. To assess the relationship between the variants identified in population and CRC development, it is very important in future research to study the interaction between genes and also the environment on the colorectal cancer risk. Although suitable for the determination of general population frequencies of independent variants, NIPT data are unsuitable for calculations (such as polygenic risk score determinations) based on exact combinations of these variants in individuals, which may de facto determine the risk of individuals to develop certain diseases.

The underlying mechanism for a high incidence of CRC in the Slovak population is still unclear at the moment; however, it is possible that genetic factors, like the most common inherited syndrome-LS, play a crucial role in colorectal etiology. We have performed a literature search in PubMed focused on population studies of CRC and LS in Slovakia from 2010-2020 using next-generation sequencing. In the Slovak population, only a few population studies of risk variants have been conducted to elucidate the etiology of CRC (55-57). In general, little is known about risk variants associated with CRC or LS in the Slovak population.

Identifying mutations associated with CRC in populations with high mortality rate, such as the Slovak population, is important to reduce the incidence of this multifactor disorder. The findings from these studies suggest a lack of understanding of the mechanism of many risk variants of CRC. Due to study limitations, we could not identify any pathogenic variants associated with LS in the Slovak population using NIPT data. On the other hand, NIPT data is not a major obstacle to better results, as pathogenic variants have extremely low frequencies in the general population. Even in most cases, the frequencies are not known. However, we identified several promising common risk variants associated with CRC previously reported in GWAS studies that represent variants with highly different frequencies between Slovak and non-Finnish populations in boxplots. Since NIPT expands rapidly to millions of individuals each year, the reuse of these data reduces the cost of large-scale population studies and likely provides an acceptable background for information about genomic variation. Finally, future population studies on larger sample sets with various types of mutations are needed to reveal new mechanisms of pathogenicity and links to new biological pathways, which may be useful in designing preventive strategies and treatment of CRC.

\section{Acknowledgements}

Not applicable.

\section{Funding}

The present study was supported by the PANGAIA project H2020-MSCA-RISE-2019 (grant no. 872539) funded under H2020-EU.1.3.3. Programme, the OP Integrated Infrastructure for the project 'Long term strategic research and development focused on the occurrence of Lynch syndrome in the Slovak population and possibilities of prevention of tumors associated with this syndrome' (grant no. 313011V578) co-financed by the European Regional Development Fund (ERDF), the Operational Program Integrated Infrastructure (grant no. 313011F988) 
co-financed by the ERDF, and the Scientific Grant Agency of the Ministry of Education, Science, Research and Sport of the Slovak Republic and the Slovak Academy of Sciences (grant no. 1/0305/19).

\section{Availability of data and materials}

The datasets generated and/or analyzed during the current study are available in the DSpace repository, https://dspace. uniba.sk/xmlui/handle/123456789/27.

\section{Authors' contributions}

NF and JG performed data analysis. NF was responsible for the literature search and manuscript writing. JG, JB and JR were responsible for designing the study and supervising the work. TS conceived the idea of the project and TS, JB and JR performed proofreading of the manuscript. JG and JB confirm the authenticity of all the raw data. All authors have read and approved the final manuscript.

\section{Ethics approval and consent to participate}

The non-invasive prenatal testing study (study ID. 35900_2015) was approved by the Ethical Committee of the Bratislava Self-Governing Region (Sabinovska ul.16, 82005 Bratislava) on 30th April 2015 (approval no. 03899_2015).

\section{Patient consent for publication}

Not applicable.

\section{Competing interests}

The authors declare that they have no competing interests.

\section{References}

1. Bray F, Ferlay J, Soerjomataram I, Siegel RL, Torre LA and Jemal A: Global cancer statistics 2018: GLOBOCAN estimates of incidence and mortality worldwide for 36 cancers in 185 countries. CA Cancer J Clin 68: 394-424, 2018.

2. Global Cancer Observatory. International Agency for Research on Cancer, Lyon, France, 2020. https://gco.iarc.fr/today/data/ factsheets/populations/703-slovakia-fact-sheets.pdf. Accessed November 9, 2020.

3. Thanikachalam K and Khan G: Colorectal cancer and nutrition. Nutrients 11: 164, 2019.

4. Rawla P, Sunkara T and Barsouk A: Epidemiology of colorectal cancer: Incidence, mortality, survival, and risk factors. Prz Gastroenterol 14: 89-103, 2019.

5. Cai S, Li Y, Ding Y, Chen K and Jin M: Alcohol drinking and the risk of colorectal cancer death: A meta-analysis. Eur J Cancer Prev 23: 532-539, 2014.

6. Dashti SG, Buchanan DD, Jayasekara H, Ouakrim DA, Clendenning M, Rosty C, Winship IM, Macrae FA, Giles GG Parry S, et al: Alcohol consumption and the risk of colorectal cancer for mismatch repair gene mutation carriers. Cancer Epidemiol Biomarkers Prev 26: 366-375, 2017.

7. Botteri E, Iodice S, Bagnardi V, Raimondi S, Lowenfels AB and Maisonneuve P: Smoking and colorectal cancer: A meta-analysis. JAMA 300: 2765-2778, 2008.

8. Limsui D, Vierkant RA, Tillmans LS, Wang AH, Weisenberger DJ, Laird PW, Lynch CF, Anderson KE, French AJ, Haile RW, et al: Cigarette smoking and colorectal cancer risk by molecularly defined subtypes. J Natl Cancer Inst 102: 1012-1022, 2010.
9. Ordóñez-Mena JM, Walter V, Schöttker B, Jenab M, O'Doherty MG, Kee F, Bueno-de-Mesquita B, Peeters PH, Stricker BH, Ruiter R, et al: Impact of prediagnostic smoking and smoking cessation on colorectal cancer prognosis: A meta-analysis of individual patient data from cohorts within the CHANCES consortium. Ann Oncol 29: 472-483, 2018.

10. Thrift AP, Gong J, Peters U, Chang-Claude J, Rudolph A, Slattery ML, Chan AT, Locke AE, Kahali B, Justice AE, et al: Mendelian randomization study of body mass index and colorectal cancer risk. Cancer Epidemiol Biomarkers Prev 24: 1024-1031, 2015.

11. Gharahkhani P, Ong JS, An J, Law MH, Whiteman DC, Neale RE and MacGregor S: Effect of increased body mass index on risk of diagnosis or death from cancer. Br J Cancer 120: 565-570, 2019.

12. Dekker E, Tanis PJ, Vleugels JLA, Kasi PM and Wallace MB: Colorectal cancer. Lancet 394: 1467-1480, 2019.

13. Lu Y, Kweon SS, Tanikawa C, Jia WH, Xiang YB, Cai Q, Zeng C, Schmit SL, Shin A, Matsuo K, et al: Large-scale genome-wide association study of east asians identifies loci associated with risk for colorectal cancer. Gastroenterology 156: 1455-1466, 2019.

14. Zeng C, Matsuda K, Jia WH, Chang J, Kweon SS, Xiang YB, Shin A, Jee SH, Kim DH, Zhang B, et al: Identification of susceptibility loci and genes for colorectal cancer risk. Gastroenterology 150: 1633-1645, 2016.

15. Al-Tassan NA, Whiffin N, Hosking FJ, Palles C, Farrington SM, Dobbins SE, Harris R, Gorman M, Tenesa A, Meyer BF, et al: A new GWAS and meta-analysis with 1000 Genomes imputation identifies novel risk variants for colorectal cancer. Sci Rep 5: 10442, 2015 .

16. Zhang B, Jia WH, Matsuda K, Kweon SS, Matsuo K, Xiang YB, Shin A, Jee SH, Kim DH, Cai Q, et al: Large-scale genetic study in East Asians identifies six new loci associated with colorectal cancer risk. Nat Genet 46: 533-542, 2014.

17. Takahashi Y, Sugimachi K, Yamamoto K, Niida A, Shimamura T, Sato T, Watanabe M, Tanaka J, Kudo S, Sugihara K, et al: Japanese genome-wide association study identifies a significant colorectal cancer susceptibility locus at chromosome $10 \mathrm{p} 14$. Cancer Sci 108: 2239-2247, 2017.

18. Schmit SL, Edlund CK, Schumacher FR, Gong J, Harrison TA, Huyghe JR, Qu C, Melas M, Van Den Berg DJ, Wang H, et al: Novel common genetic susceptibility loci for colorectal cancer. J Natl Cancer Inst 111: 146-157, 2019.

19. Biller LH, Syngal S and Yurgelun MB: Recent advances in lynch syndrome. Fam Cancer 18: 211-219, 2019.

20. Yurgelun MB and Hampel H: Recent advances in lynch syndrome: Diagnosis, treatment, and cancer prevention. Am Soc Clin Oncol Educ Book 38: 101-109, 2018.

21. Møller P, Seppälä T, Bernstein I, Holinski-Feder E, Sala P, Evans DG, Lindblom A, Macrae F, Blanco I, Sijmons R, et al: Incidence of and survival after subsequent cancers in carriers of pathogenic MMR variants with previous cancer: A report from the prospective lynch syndrome database. Gut 66: 1657-1664, 2017.

22. Møller P, Seppälä TT, Bernstein I, Holinski-Feder E, Sala P, Evans DG, Lindblom A, Macrae F, Blanco I, Sijmons RH, et al: Cancer risk and survival in carriers by gene and gender up to 75 years of age: A report from the prospective lynch syndrome database. Gut 67: 1306-1316, 2018.

23. Soares BL, Brant AC, Gomes R, Pastor T, Schneider NB, Ribeiro-Dos-Santos Â, de Assumpção PP, Achatz MI, Ashton-Prolla P and Moreira MA: Screening for germline mutations in mismatch repair genes in patients with lynch syndrome by next generation sequencing. Fam Cancer 17: 387-394, 2018.

24. Cox VL, Bamashmos AA, Foo WC, Gupta S, Yedururi S, Garg N and Kang HC: Lynch syndrome: Genomics update and imaging review. Radiographics 38: 483-499, 2018.

25. Le S, Ansari U, Mumtaz A, Malik K, Patel P, Doyle A and Khachemoune A: Lynch syndrome and muir-torre syndrome: An update and review on the genetics, epidemiology, and management of two related disorders. Dermatol Online J 23: 13030, 2017.

26. Peltomäki P: Update on lynch syndrome genomics. Fam Cancer 15: 385-393, 2016.

27. Duraturo F, Liccardo R, Cavallo A, De Rosa M, Grosso M and Izzo P: Association of low-risk MSH3 and MSH2 variant alleles with Lynch syndrome: Probability of synergistic effects. Int J Cancer 129: 1643-1650, 2011.

28. Kuiper RP, Vissers LELM, Venkatachalam R, Bodmer D, Hoenselaar E, Goossens M, Haufe A, Kamping E, Niessen RC, Hogervorst FB, et al: Recurrence and variability of germline EPCAM deletions in Lynch syndrome. Hum Mutat 32: 407-414, 2011. 
29. Shah SN, Hile SE and Eckert KA: Defective mismatch repair, microsatellite mutation bias, and variability in clinical cancer phenotypes. Cancer Res 70: 431-435, 2010.

30. Martin-Morales L, Rofes P, Diaz-Rubio E, Llovet P, Lorca V, Bando I, Perez-Segura P, de la Hoya M, Garre P, Garcia-Barberan $\mathrm{V}$ and Caldes T: Novel genetic mutations detected by multigene panel are associated with hereditary colorectal cancer predisposition. PLoS One 13: e0203885, 2018.

31. Budis J, Gazdarica J, Radvanszky J, Harsanyova M, Gazdaricova I, Strieskova L, Frno R, Duris F, Minarik G, Sekelska M, et al: Non-invasive prenatal testing as a valuable source of population specific allelic frequencies. J Biotechnol 299: 72-78, 2019.

32. Pös O, Budis J, Kubiritova Z, Kucharik M, Duris F, Radvanszky J and Szemes T: Identification of structural variation from NGS-Based non-invasive prenatal testing. Int J Mol Sci 20: 4403, 2019.

33. Liu S, Huang S, Chen F, Zhao L, Yuan Y, Francis SS, Fang L, Li Z, Lin L, Liu R, et al: Genomic analyses from non-invasive prenatal testing reveal genetic associations, patterns of viral infections, and Chinese population history. Cell 175: 347-359, 2018.

34. Tran NH, Vo TB, Nguyen VT, Tran NT, Trinh THN, Pham HAT, Dao THT, Nguyen NM, Van YLT, Tran VU, et al: Genetic profiling of Vietnamese population from large-scale genomic analysis of non-invasive prenatal testing data. Sci Rep 10: 19142, 2020.

35. Pös $\mathrm{O}$, Budiš $\mathbf{J}$ and Szemes $\mathrm{T}$ : Recent trends in prenatal genetic screening and testing. F1000Res 8: F1000, 2019.

36. Minarik G, Repiska G, Hyblova M, Nagyova E, Soltys K, Budis J, Duris F, Sysak R, Bujalkova MG, Vlkova-Izrael B, et al: Utilization of benchtop next generation sequencing platforms ion torrent PGM and MiSeq in noninvasive prenatal testing for chromosome 21 trisomy and testing of impact of in silico and physical size selection on its analytical performance. PLoS One 10: e0144811, 2015.

37. Beyene J and Pare G. Statistical genetics with application to population-based study design: A primer for clinicians. Eur Heart J 35: 495-500, 2014.

38. Zhu L, Huang Y, Fang X, Liu C, Deng W, Zhong C, Xu J, Xu D and Yuan Y: A novel and reliable method to detect microsatellite instability in colorectal cancer by next-generation sequencing. J Mol Diagn 20: 225-231, 2018.

39. Yurgelun MB, Allen B, Kaldate RR, Bowles KR, Judkins T, Kaushik P, Roa BB, Wenstrup RJ, Hartman AR and Syngal S: Identification of a variety of mutations in cancer predisposition genes in patients with suspected lynch syndrome. Gastroenterology 149: 604-613, 2015

40. Valle L, de Voer RM, Goldberg Y, Sjursen W, Försti A, Ruiz-Ponte C, Caldés T, Garré P, Olsen MF, Nordling M, et al: Update on genetic predisposition to colorectal cancer and polyposis. Mol Aspects Med 69: 10-26, 2019.

41. Budiš J, Kucharík M, Duriš F, Gazdarica J, Zrubcová M, Ficek A, Szemes T, Brejová B and Radvanszky J: Dante: Genotyping of known complex and expanded short tandem repeats. Bioinformatics 35: 1310-1317, 2019.

42. Jiao S, Peters U, Berndt S, Brenner H, Butterbach K, Caan BJ, Carlson CS, Chan AT, Chang-Claude J, Chanock S, et al: Estimating the heritability of colorectal cancer. Hum Mol Genet 23: 3898-3905, 2014.

43. Law PJ, Timofeeva M, Fernandez-Rozadilla C, Broderick P, Studd J, Fernandez-Tajes J, Farrington S, Svinti V, Palles C, Orlando G, et al: Association analyses identify 31 new risk loci for colorectal cancer susceptibility. Nat Commun 10: 2154, 2019.

44. Zhang K, Civan J, Mukherjee S, Patel F and Yang H: Genetic variations in colorectal cancer risk and clinical outcome. World J Gastroenterol 20: 4167-4177, 2014.

45. Hofer P, Hagmann M, Brezina S, Dolejsi E, Mach K, Leeb G, Baierl A, Buch S, Sutterlüty-Fall H, Karner-Hanusch J, et al: Bayesian and frequentist analysis of an Austrian genome-wide association study of colorectal cancer and advanced adenomas. Oncotarget 8: 98623-98634, 2017.

46. Wang H, Schmit SL, Haiman CA, Keku TO, Kato I, Palmer JR, van den Berg D, Wilkins LR, Burnett T, Conti DV, et al. Nove colon cancer susceptibility variants identified from a genomewide association study in African Americans. Int J Cancer. 140: 2728-2733, 2017.

47. Closa A, Cordero D, Sanz-Pamplona R, Solé X, Crous-Bou M, Paré-Brunet L, Berenguer A, Guino E, Lopez-Doriga A, Guardiola J, et al: Identification of candidate susceptibility genes for colorectal cancer through eQTL analysis. Carcinogenesis 35: 2039-2046, 2014.
48. Dunlop MG, Dobbins SE, Farrington SM, Jones AM, Palles C, Whiffin N, Tenesa A, Spain S, Broderick P, Ooi LY, et al: Common variation near CDKN1A, POLD3 and SHROOM2 influences colorectal cancer risk. Nat Genet 44: 770-776, 2012.

49. Wang H, Haiman CA, Burnett T, Fortini BK, Kolonel LN, Henderson BE, Signorello LB, Blot WJ, Keku TO, Berndt SI, et al: Fine-mapping of genome-wide association study-identified risk loci for colorectal cancer in African Americans. Hum Mol Genet 22: 5048-5055, 2013.

50. Hong SN, Park C, Kim JI, Kim DH, Kim HC, Chang DK, Rhee PL, Kim JJ, Rhee JC, Son HJ and Kim YH: Colorectal cancer-susceptibility single-nucleotide polymorphisms in Korean population. J Gastroenterol Hepatol 30: 849-857, 2015.

51. Moazeni-Roodi A, Ghavami S, Ansari H and Hashemi M: Association between the flap endonuclease 1 gene polymorphisms and cancer susceptibility: An updated meta-analysis. J Cell Biochem 120: 13583-13597, 2019.

52. Chou AK, Shen MY, Chen FY, Hsiao CL, Shih LC, Chang WS, Tsai CW, Ying TH, Wu MH, Huang CY and Bau DT: The association of flap endonuclease 1 genotypes with the susceptibility of endometriosis. Cancer Genomics Proteomics 14: 455-460, 2017.

53. Kubiritova Z, Gyuraszova M, Nagyova E, Hyblova M, Harsanyova M, Budis J, Hekel R, Gazdarica J, Duris F, Kadasi L, et al: On the critical evaluation and confirmation of germline sequence variants identified using massively parallel sequencing. J Biotechnol 298: 64-75, 2019.

54. Trost B, Engchuan W, Nguyen CM, Thiruvahindrapuram B, Dolzhenko E, Backstrom I, Mirceta M, Mojarad BA, Yin Y, Dov A, et al: Genome-wide detection of tandem DNA repeats that are expanded in autism. Nature 586: 80-86, 2020.

55. Mahmood S, Sivoňová M, Matáková T, Dobrota D, Wsólová L, Dzian A, et al: Association of EGF and p53 gene polymorphisms and colorectal cancer risk in the Slovak population. Cent Eur J Med 9: 405-416, 2014

56. Škereňová M, Halašová E, Matáková T, Jesenská L, Jurečeková J, Šarlinová M, Čierny D and Dobrota D: Low variability and stable frequency of common haplotypes of the tp53 gene region in colorectal cancer patients in a Slovak population. Anticancer Res 37: 1901-1907, 2017.

57. Kašubová I, Kalman M, Jašek K, Burjanivová T, Malicherová B, Vaňochová A, Meršaková S, Lasabová Z and Plank L: Stratification of patients with colorectal cancer without the recorded family history. Oncol Lett 17: 3649-3656, 2019.

58. Jia WH, Zhang B, Matsuo K, Shin A, Xiang YB, Jee SH, Kim DH, Ren Z, Cai Q, Long J, et al: Genome-wide association analyses in East Asians identify new susceptibility loci for colorectal cancer. Nat Genet 45: 191-196, 2013.

59. Peters U, Jiao S, Schumacher FR, Hutter CM, Aragaki AK, Baron JA, Berndt SI, Bézieau S, Brenner H, Butterbach K, et al: Identification of genetic susceptibility loci for colorectal tumors in a genome-wide meta-analysis. Gastroenterology 144: 799-807, 2013.

60. Whiffin N, Hosking FJ, Farrington SM, Palles C, Dobbins SE, Zgaga L, Lloyd A, Kinnersley B, Gorman M, Tenes A, et al: Identification of susceptibility loci for colorectal cancer in a genome-wide meta-analysis. Hum Mol Genet 23: 4729-4737, 2014.

61. Houlston RS, Cheadle J, Dobbins SE, Tenesa A, Jones AM, Howarth K, Spain SL, Broderick P, Domingo E, Farrington S, et al: Meta-analysis of three genome-wide association studies identifies susceptibility loci for colorectal cancer at 1q41,3q26.2, 12q13.13 and 20q13.33. Nat Genet 42: 973-977, 2010.

62. Orlando G, Law PJ, Palin K, Tuupanen S, Gylfe A, Hänninen UA, Cajuso T, Tanskanen T, Kondelin J, Kaasinen E, et al: Variation at 2q35 (PNKD and TMBIM1) influences colorectal cancer risk and identifies a pleiotropic effect with inflammatory bowel disease. Hum Mol Genet 25: 2349-2359, 2016.

63. Schmit SL, Schumacher FR, Edlund CK, Conti DV, Raskin L, Lejbkowicz F, Pinchev M, Rennert HS, Jenkins MA, Hopper JL, et al: A novel colorectal cancer risk locus at 4q32.2 identified from an international genome-wide association study. Carcinogenesis 35: 2512-2519, 2014.

64. Peters U, Hutter CM, Hsu L, Schumacher FR, Conti DV, Carlson CS, Edlund CK, Haile RW, Gallinger S, Zanke BW, et al: Meta-analysis of new genome-wide association studies of colorectal cancer risk. Hum Genet 131: 217-234, 2012.

65. Tomlinson IPM, Webb E, Carvajal-Carmona L, Broderick P, Howarth K, Pittman AM, Spain S, Lubbe S, Walther A, Sullivan K, et al: A genome-wide association study identifies colorectal cancer susceptibility loci on chromosomes 10p14 and 8q23.3. Nat Genet 40: 623-630, 2008 . 
66. Tenesa A, Farrington SM, Prendergast JGD, Porteous ME, Walker M, Haq N, Barnetson RA, Theodoratou E, Cetnarskyj R, Cartwright $\mathrm{N}$, et al: Genome-wide association scan identifies a colorectal cancer susceptibility locus on 11q23 and replicates risk loci at 8q24 and 18q21. Nat Genet 40: 631-637, 2008.

67. Tomlinson IPM, Carvajal-Carmona LG, Dobbins SE, Tenesa A, Jones AM, Howarth K, Palles C, Broderick P, Jaeger EEM, Farrington S, et al: Multiple common susceptibility variants near BMP pathway loci GREM1, BMP4, and BMP2 explain part of the missing heritability of colorectal cancer. PLoS Genet 7: e1002105, 2011.

68. COGENT Study; Houlston RS, Webb E, Broderick P,Pittman AM, Di Bernardo MC, Lubbe S, Chandler I, Vijayakrishnan J, Sullivan K, et al: Meta-analysis of genome-wide association data identifies four new susceptibility loci for colorectal cancer. Nat Genet 40: 1426-1435, 2008.

69. Broderick P, Carvajal-Carmona L, Pittman AM, Webb E, Howarth K, Rowan A, Lubbe S, Spain S, Sullivan K, Fielding S, et al: A genome-wide association study shows that common alleles of SMAD7 influence colorectal cancer risk. Nat Genet 39: 1315-1317, 2007.

70. Schumacher FR, Schmit SL, Jiao S, Edlund CK, Wang H, Zhang B, Hsu L, Huang SC, Fischer CP, Harju JF, et al: Genome-wide association study of colorectal cancer identifies six new susceptibility loci. Nat Commun 6: 7138, 2015.

71. Haiman CA, Le Marchand L, Yamamato J, Stram DO, Sheng X, Kolonel LN, Wu AH, Reich D and Henderson BE: A common genetic risk factor for colorectal and prostate cancer. Nat Genet 39: 954-956, 2007.
72. Tomlinson I, Webb E, Carvajal-Carmona L, Broderick P, Kemp Z, Spain S, Penegar S, Chandler I, Gorman M, Wood W, et al: A genome-wide association scan of tag SNPs identifies a susceptibility variant for colorectal cancer at 8q24.21. Nat Genet 39: 984-988, 2007.

73. Hutter CM, Slattery ML, Duggan DJ, Muehling J, Curtin K, Hsu L, Beresford SA, Rajkovic A, Sarto GE, Marshall JR, et al: Characterization of the association between 8q24 and colon cancer: Gene-environment exploration and meta-analysis. BMC Cancer 10: 670, 2010.

74. Cui R, Okada Y, Jang SG, Ku JL, Park JG, Kamatani Y, Hosono N, Tsunoda T, Kumar V, Tanikawa C, et al: Common variant in 6q26-q27 is associated with distal colon cancer in an Asian population. Gut 60: 799-805, 2011.

75. Wang H, Burnett $T$, Kono S, Haiman CA, Iwasaki M, Wilkens LR, Loo LW, Van Den Berg D, Kolonel LN, Henderson BE, et al: Transethnic genome-wide association study of colorectal cancer identifies a new susceptibility locus in VTI1A. Nat Commun 5: 4613, 2014.

This work is licensed under a Creative Commons Attribution-NonCommercial-NoDerivatives 4.0 International (CC BY-NC-ND 4.0) License. 